\title{
The sentence completion test for depression can distinguish between people with and without major depressive disorder
}

Barton S, Morley S, Bloxham G, et al. Sentence completion test for depression (SCD): an idiographic measure of depressive thinking. Br J Clin Psychol 2005;44:29-46.

Does the sentence completion test for depression differentiate between people with and people without depression?

METHODS

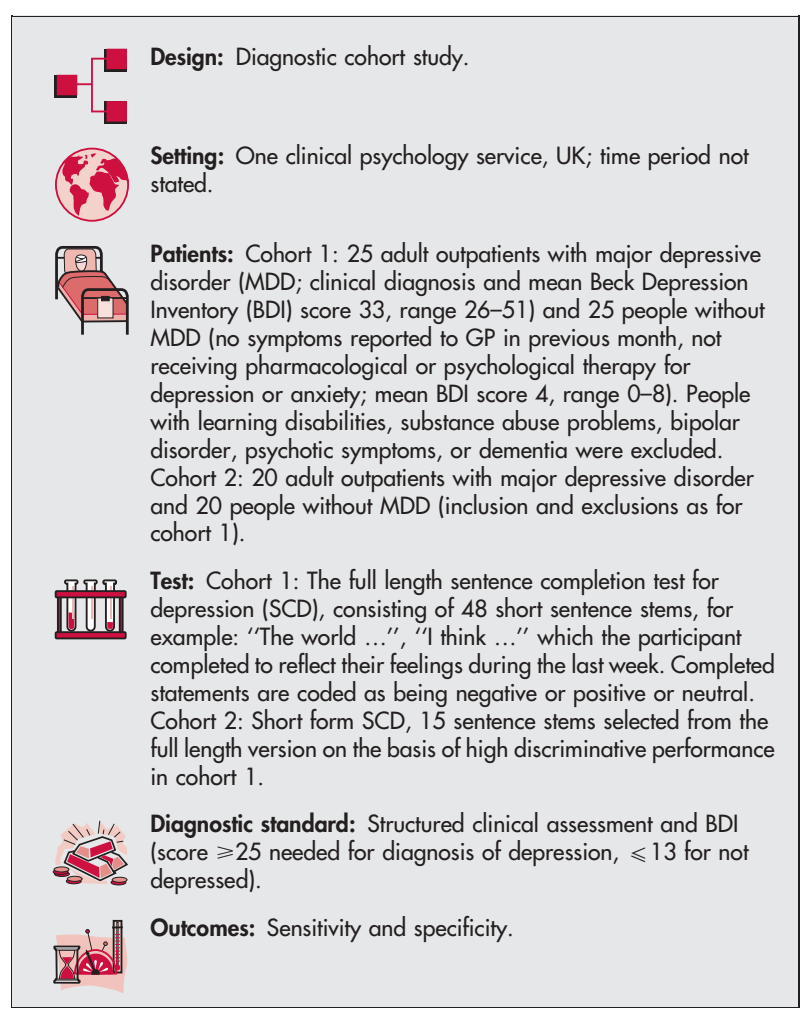

\section{MAIN RESULTS}

For the full length sentence completion test for depression (SCD), using a cut off score of 16 negative completions for a diagnosis of depression gave a sensitivity of $92 \%$ and a specificity of $96 \%$. The short form SCD had a sensitivity and specificity of $100 \%$ when used with a cut off score of 5 or more negative completions.

\section{CONCLUSIONS}

The full length and short forms of the SCD show high sensitivity and specificity for the differentiation between people with and without clinically diagnosed major depressive disorder.

For correspondence: Dr Stephen Barton, School of Neuroscience and Psychiatry, University of Newcastle, Ridley Building, Newcastle Upon Tyne NE1 7RU, UK; s.b.barton@ncl.ac.uk

Sources of funding: none reported.

\section{NOTES}

One limitation of the study may be the use of clinical assessment rather than a research diagnostic interview. In addition, estimates of sensitivity and specificity may not be representative of what might be achieved in the general population, due to the use of two well defined comparator groups, with non-contiguous ranges of BDI scores.

\section{Commentary}

A multiplicity of validated self report depression questionnaires is available both for research and medical care. The study by Barton et $a$ is innovative because it investigates the reliability and validity of a completely different type of self report questionnaire, the sentence completion test for depression (SCD). Whereas conventional self report questionnaires assess the presence of various depression symptoms using preset response categories, ${ }^{1}$ the SCD includes 48 brief sentence stems (short form: 15 sentence stems), and the patient is asked to provide continuations to the incomplete sentences. The total score of the SCD is assessed by adding the number of negative and positive completions.

Based on the results of three studies, the authors report good internal consistency, interrater reliability, construct validity, criterion validity, and sensitivity to change for the SCD. However some methodological limitations deserve mentioning: the sample sizes for all three studies were small ( $n=50, n=40$, and $n=18$, respectively), only patients with either very high or very low depression scores were included $(B D I \geqslant 25$, or $\mathrm{BDI} \leqslant 13$, respectively), and no validated diagnostic interview was used as the criterion standard for depressive disorder.

The clinical value of the SCD is that it gives specific information on the patients' attitudes towards themselves, their interpersonal relationships, the world, the future, and the past. This information is particularly useful for the mental health professional treating someone with depression. Given the preliminary psychometric properties of the SCD, the low level of standardisation, and the relatively high expenditure of time for both patient and mental health professional, the SCD will not supersede conventional self report depression questionnaires. For case finding, measuring depression severity, establishing depression diagnosis, and monitoring response to treatment, brief conventional questionnaires with preset response categories ${ }^{2} 3$ seem more practical and efficient. Altogether, the SCD appears as an ideographic depression measure that is clinically promising when used complementary to conventional self report depression questionnaires.

Bernd Löwe, MD, PhD

University of Heidelberg, Department of Psychosomatic and General Internal Medicine, Heidelberg, Germany

1 Williams JW, Pignone $M$, Ramirez $G$, et al. Identifying depression in primary care: a literature synthesis of case-finding instruments. Gen Hosp Psychiatry 2002;24:225-37.

2 Spitzer RL, Kroenke K, Williams JB, Patient Health Questionnaire Primary Care Study Group. Validation and utility of a self-report version of PRIMEMD: The PHQ primary care study. JAMA 1999;282:1737-44.

3 Löwe B, Kroenke K, Gräfe K. Detecting and monitoring depression with a 2-item questionnaire (PHQ-2). J Psychosom Res 2005;58:163-71. 